\title{
A SIMULATOR OF THE HUMAN HEAD ROTATION
}

\section{SKULA, D[avid]}

Abstract: The article deals with design of a device for simulation of human head rotation. This device is used for testing sensors of angular rotation which are used for measuring human head rotation. Firstly, parameters of human head rotation are discussed. Then hardware is chosen based on these parameters. At the end communication with tested sensors is solved

Key words: head rotation, simulator, inertial sensors

\section{INTRODUCTION}

The idea to create a device to simulate the rotation of a human head arose from the requirement to select a sensor to detect head rotation in virtual reality glasses. Rotation of an operator head is transferred to rotation of a camera on a robot. The requirements satisfy accelerometers, gyroscopes and magnetometers. Commercially available inertial measurement units (IMU) include a combination of these sensors together with the evaluation. Most of them are designed more for the inertial navigation. Measurement of human head rotation is slightly different.

The main differences are:

-Wide dynamic range of measurement. From very slow movements to very rapid changes in the head position.

-The absence of translational motion.

It is obvious that not every sensor of angular rotation is suitable for the measurement of human head rotation. The device for simulating human head rotation gives a tool for testing and comparing different types of sensors and different types of evaluation algorithms.

\section{PARAMETERS OF HUMAN HEAD ROTATION}

Before we start designed the simulator, we have to know the basic parameters of human head rotation. The most important parameter is a maximal angular acceleration that a human head can achieve.

We designed an experiment to determine the maximal angular acceleration of the human head. We installed a rotation encoder on a helmet and done some measurements. Results one of these measurements is on Fig. 1. A person looking left and right as fast as he could during the measurement.

First graph on Fig. 1 represents angle of rotation, second graph angular velocity and last graph angular acceleration. Angular velocity was obtained derivation of rotation angle and angular acceleration derivation of angular velocity.

The Fig. 1 shows that the maximal angular acceleration is $40000 \% \mathrm{~s}^{2}$. The simulator should therefore be able to achieve such acceleration.
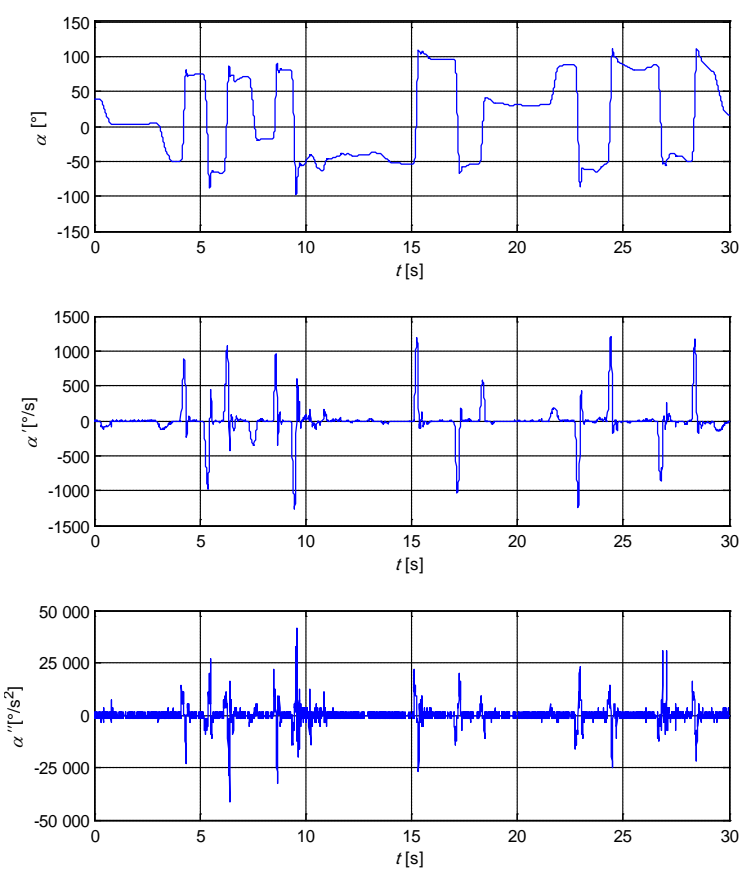

Fig. 1. A Measurement of Human Head Rotation

\section{THE HARDWARE DESIGN OF THE SIMULATOR}

The movement of the human head is very specific issue. The dynamic range of motion is large and to describe the rotation of the human head is required three-axis rotation system. If we increase dynamic range of the device, the sizes of required motors increase too and cost of three-axis rotation system increases rapidly.

Therefore, we chose two-axis rotation system. One axis represents head movement by looking left and right. Second axis represents head movement by looking up and down. It is simplification but sufficient. The camera on the robot is usually mounted on two axes. The block scheme of the simulator is on Fig. 2.

Motor M1 simulates head movement by looking up and down and motor M2 simulates head movement by looking left and right.

The dynamic of the motor M2 is not a big problem. Sensors weight is almost negligible. The engine must satisfy dynamic demands only without load on the shaft. Motor TGH3-0160 (TG drives) was chosen. It weighs $2,6 \mathrm{~kg}$, dimensions are $0,127 \times 0,08 \mathrm{~m}$ and nominal torque is $1,5 \mathrm{Nm}$.

The shaft of motor M1 carries the full weight of motor M2 and construction around this motor. Weight of this construction and the motor M2 is approximately $m=4 \mathrm{~kg}$ and the dimensions can be considered as cylindrical with the base 
radius $R=0,05 \mathrm{~m}$ and the length $L=0,14 \mathrm{~m}$. Maximal angular acceleration is $\alpha^{\prime \prime}=40000 \% \mathrm{~s}^{2}=698 \mathrm{rad} / \mathrm{s}^{2}$ and construction offset is $h=0,03 \mathrm{~m}$. Nominal torque of motor M1 is then $M=8,8 \mathrm{Nm}(1)$.

$$
M=\left(\frac{1}{4} m R^{2}+\frac{1}{12} m L^{2}+m h^{2}\right) \cdot \alpha^{\prime \prime}
$$

Nominal torque of selected motor 115MSC200TAAAA (Dutymax) is $10 \mathrm{Nm}$ which is enough.

The shaft of motor M1 is equipped with a precise rotation encoder (E) and a torque overload protection (T).

Control is provided by inverters. Inverter TGA-24-9/20 controls motor M2 and inverter UNI1405 controls motor M1. All electronics (inverters, EMI filters, circuit breakers ...) is located in the distribution board.

The simulator allows very precise adjustment of the angle in horizontal axis (precise encoder E) and adjustment of the angle in vertical axis (motor M2 includes an encoder).

Some sensors have to be measured during continuous rotation to determine some parameters (gyroscopes for example). Sensors are battery powered and wirelessly measured (chapter IV) so continual rotating is possible in the vertical axis.

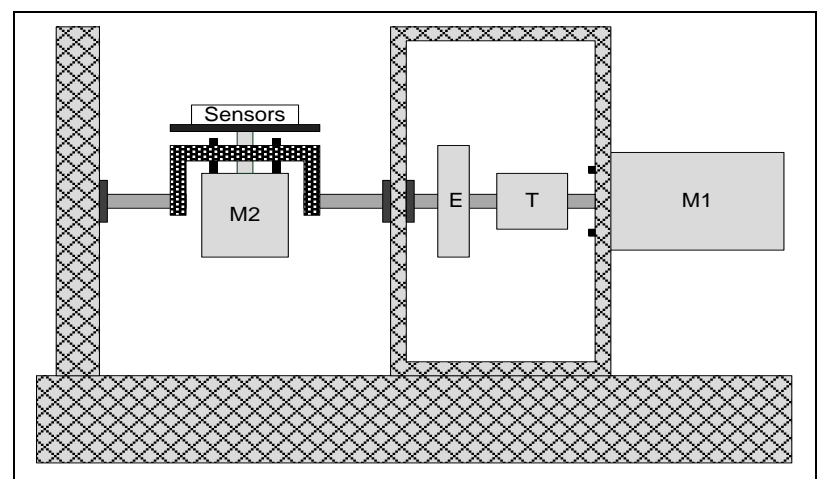

Fig. 2. A Mechanical Design of the Simulator

\section{COMMUNICATION WITH TESTED SENSORS}

Tested sensors are battery powered and transmitted wirelessly to a PC (Fig. 3).

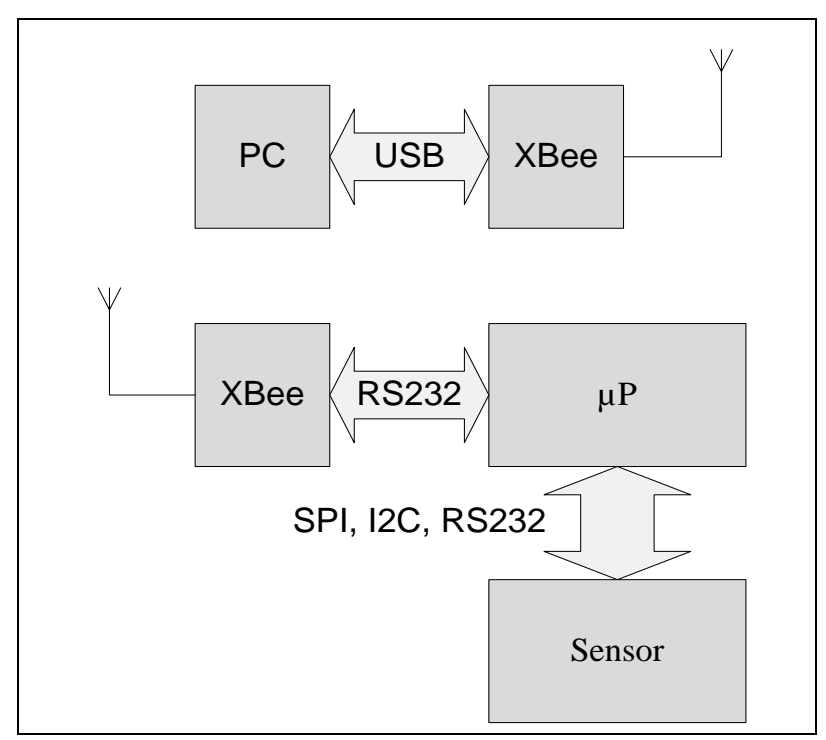

Fig. 3. A Block Scheme of Communication with Tested Sensors
Most of the sensors communicate via a standard communication bus. The simulator is equipped with microprocessor which can be connected to the sensor with SPI, I2C or RS232. Measured data from sensor are sent via RS232 to the wireless module XBee ZigBee.

On the PC side, data are sent to the PC via USB bus.

\section{CONCLUSION}

The result is a device for simulating a human head rotation. The dynamic of the device is sufficiently close to the real movements of the head. The simulator allows adjustment of the angle in two axes. First axis represents head movement by looking left and right. Second axis represents head movement by looking up and down. It is simplification but sufficient. Testing sensors during continuous rotation is possible in one axis (vertical axis).

Data from sensors are transmitted wirelessly to a PC.

\section{ACKNOWLEDGMENT}

This work has been supported in part by Ministry of Education, Youth and Sports of the Czech Republic (Research Intent MSM0021630529 Intelligent systems in automation), Grant Agency of the Czech Republic (102/09/H081 SYNERGY - Mobile Sensoric Systems and Network) and by Brno University of Technology.

This work was supported by grant „Modern Methods and Approaches in Automation“ from the Internal Grant Agency of Brno University of Technology (grant No. FEKT-S-10-12).

\section{REFERENCES}

Titterton, F. H., Weston, J. L. (2004) Strapdown Inertial Navigation Technology. Institution of Engineering and Technology, 581pages, ISBN 1-56347-693-2

*** (2011) Digital servoamplifier TGA-24-9/20 Instruction manual, TG Drives, Available from:

http://www.tgdrives.cz/fileadmin/user_upload/download/ser vopohony_tga/EN_TGA_HW_manual_03_04.pdf, Accessed on: 2011-08-20

*** (2005) Digital servoamplifier TGA-24-9/20 Description of the set up softwaqre S.C.D., TG Drives, Available from: http://www.tgdrives.cz/fileadmin/user_upload/download/ser vopohony_tga/Soft-manual_05_05_en.pdf, Accesed on: 2011-08-20

*** (2005) Digital servoamplifier TGA-24-9/20 Registers description, TG Drives, Available from:

http://www.tgdrives.cz/fileadmin/user_upload/download/ser vopohony_tga/Registry288-en.pdf, Accesed on: 2011-08-20

*** (2000) Uživatelská příručka měniče kmitočtu řady UNIDRIVE, Control Techniques

*** (1999) UD53 Resolver interface small option module for Unidrive, Control Techniques

*** (2008) XBee Multipoint RF modules, Digi Internation Inc., Available from:

http://www.digi.com/pdf/ds_xbeemultipointmodules.pdf, Accesed on: 2011-08-20 